\title{
In silico evolutionary analysis of Helicobacter pylori outer membrane phospholipase A (OMPLA)
}

Hilde S Vollan ${ }^{1 *}$, Tone Tannæs ${ }^{1}$, Yoshio Yamaoka² and Geir Bukholm³,4

\begin{abstract}
Background: In the past decade, researchers have proposed that the pldA gene for outer membrane phospholipase A (OMPLA) is important for bacterial colonization of the human gastric ventricle. Several conserved Helicobacter pylori genes have distinct genotypes in different parts of the world, biogeographic patterns that can be analyzed through phylogenetic trees. The current study will shed light on the importance of the pldA gene in $H$. pylori. In silico sequence analysis will be used to investigate whether the bacteria are in the process of preserving, optimizing, or rejecting the pldA gene. The pldA gene will be phylogenetically compared to other housekeeping (HK) genes, and a possible origin via horizontal gene transfer (HGT) will be evaluated through both intra- and interspecies evolutionary analyses.

Results: In this study, pldA gene sequences were phylogenetically analyzed and compared with a large reference set of concatenated HK gene sequences. A total of 246 pldA nucleotide sequences were used; 207 were from Norwegian isolates, 20 were from Korean isolates, and 19 were from the NCBI database. Best-fit evolutionary models were determined with MEGA5 ModelTest for the pldA $(K 80+I+G)$ and HK $(G T R+I+G)$ sequences, and maximum likelihood trees were constructed. Both HK and pldA genes showed biogeographic clustering. Horizontal gene transfer was inferred based on significantly different GC contents, the codon adaptation index, and a phylogenetic conflict between a tree of OMPLA protein sequences representing 171 species and a tree of the AtpA HK protein for 169 species. Although a vast majority of the residues in OMPLA were predicted to be under purifying selection, sites undergoing positive selection were also found.

Conclusions: Our findings indicate that the pldA gene could have been more recently acquired than seven of the HK genes found in $\mathrm{H}$. pylori. However, the common biogeographic patterns of both the HK and pldA sequences indicated that the transfer occurred long ago. Our results indicate that the bacterium is preserving the function of OMPLA, although some sites are still being evolutionarily optimized.
\end{abstract}

Keywords: OMPLA, pldA, Phospholipase A, Outer membrane, Helicobacter pylori, Biogeography, Adaptation

\section{Background}

More than half of the world's population is colonized with Helicobacter pylori [1]. Colonization usually occurs in early childhood and results in disease in about $10 \%$ of cases [2]. This disease will in most cases be diagnosed as gastric or duodenal ulcers, while some cases will be diagnosed as gastric cancer [3].

The human gastric ventricle is the only known natural habitat for H. pylori, and one bacterial strain usually

\footnotetext{
* Correspondence: h.s.vollan@medisin.uio.no

'Department of Clinical Molecular Biology and Laboratory Sciences (EpiGen), Division of Medicine, Akershus University Hospital, University of Oslo, Oslo, Norway

Full list of author information is available at the end of the article
}

establishes a chronic, lifelong, persistent colonization in one individual [4]. Helicobacter pylori has a high level of sequence variation and has therefore been referred to as a quasi-species [5-7]. Natural transformation by exogenous DNA $[8,9]$, mutations, and recombinations are probably important mechanisms for $H$. pylori adaption and survival; for example, a variable genome could give advantages in evading the host's immune system. In spite of the high sequence variation observed in $H$. pylori, 1237 core genes have been described that are common to the analyzed $H$. pylori genomes. The amino acid identities range between $65-100 \%$. Among these core genes are housekeeping $(\mathrm{HK})$ genes that are essential for $H$. pylori survival, and the genetic variability in these genes remains very low

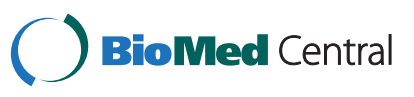


$[10,11]$. This conservation is reflected in phylogenetic analysis, where HK genes have been used to trace human migration, indicating co-evolution between $H$. pylori and its host. Linz et al. traced $H$. pylori infection in humans to before their migration from Africa through sequence analysis $[11,12]$.

Analyses of conserved $H$. pylori genes indicate the evolution of distinct genotypes in different parts of the world. The virulence factor cytotoxin-associated gene A protein, CagA, shows biogeographic variation. Yamaoka et al. postulated that the geographical differences that are observed in the incidence of gastric cancer could be explained by different $H$. pylori strains (with regard to the distribution of $\operatorname{cag} A$ and $v a c A$ genotype) [13]. CagA is injected in the host cell through the Type IV secretion system (T4SS) which is coded by Cag Pathogenicity Island (cagPAI) genes. These genes are also involved in horizontal gene transfer (HGT). Genes integrated into the $H$. pylori genome via HGT may have originated from either other bacteria or eukaryotic cells [14]. Olbermann et al. [15] analyzed the selection pressure for cagPAI genes and found that one-third of the genes were under positive selection. Most of the genes under positive selection, including the $\operatorname{cag} A$ gene, code for surface-exposed proteins. In positive selection, mutations increase fitness and, thus, new alleles increase in frequency in the population. In neutral (or nearly neutral) selection, mutations have no drastic effect on fitness and increase or decrease in frequency by chance. When fitness decreases due to deleterious mutations, new alleles are removed through purifying selection (i.e. virD4 and virB11 found in T4SS) [15].

Several authors have proposed that the pldA gene (coding for outer membrane phospholipase A, OMPLA) is important for the ability of the bacterium to colonize the human gastric ventricle [16,17]. Tannæs et al. [18] characterized a classical phase-variation in this gene due to DNA slippage in a homopolymeric tract that results in either a complete ( $p l d A \mathrm{ON})$ or truncated protein (pldAOFF). The homopolymeric tract was found in all of the clinical isolates of $H$. pylori sequenced by Tannæs et al. [18]. The conservation of the homopolymeric tract in this gene through phylogenesis underlines the importance of the gene product and maintenance of the phase variation for this bacterium. This study investigated the evolution of the pldA gene in $H$. pylori. In silico sequence analysis was used to determine whether the bacteria were in the process of preserving, optimizing, or perhaps even rejecting the $p l d A$ gene. Sequences of $p l d A$ were compared by both identity and phylogenetic analysis to a reference set of HK genes from a large number of isolates sequenced by Falush et al. [11]. Horizontal gene transfer prediction was carried out via both intraand inter-species phylogenetic analysis using related taxa and the estimation of both codon bias and GC content in $H$. pylori isolates.

\section{Results}

\section{CagA EPIYA genotyping}

All of the 20 Korean sequences had an East Asian cagA ABD genotype. Nearly all of the 50 isolates analyzed from Norway had Western cagA genotypes, with the following distribution: $66 \% \mathrm{ABC}, 12 \% \mathrm{ABCC}, 12 \% \mathrm{AB}, 4 \%$ $\mathrm{ABCCC}$, and $2 \% \mathrm{AC}$. The two isolates collected from patients with East Asian origins displayed a cagA ABD genotype (4\%).

\section{Amplification of vacA}

One Norwegian isolate from a patient of North-African origin was VacA genotyped. The sample contained an $\mathrm{s} 1 \mathrm{~b}$ allele and the $\mathrm{m} 1 \mathrm{mid}$-region type.

\section{Bioinformatic analyses of $H$. pylori pldA and seven core housekeeping genes}

Gene evolution was assessed by comparing $H$. pylori pldA gene sequences to concatenated core $\mathrm{HK}$ genes. The average pairwise sequence identity was $97.26 \% \pm$ 0.01 for the pldA sequences and $95.60 \% \pm 0.01$ for the HK genes. The average genetic distance of the pldA genes was 0.03 , while it was 0.05 for the concatenated HK genes.

The phylogenetic reference tree of concatenated $\mathrm{HK}$ genes is shown in Figure 1. With a few exceptions, the sequences clustered as expected according to geographic region. In this phylogenetic tree, the majority of sequences were from European isolates. They were separated into two clades by the African and East Asian isolates. The East Asian cluster could be further subdivided into Maorian, East Asian, and Amerindian sequences. Two isolates collected in Norway grouped in the East Asian subcluster; these patients were of East Asian origin. As expected, the remaining two samples originating from Norway were found in the European cluster in the reference tree. Pecan4 was isolated from a Peruvian patient and thus initially classified as an Amerindian strain, however, it does not cluster with the other Amerindians in the East Asian cluster as was observed by Kawi et al. [19]. Two isolates in our tree were described by Falush as hpAfrica but clustered with European sequences, and both patients were Cape Colored or Mezito, with European ancestors. Four outliers were not found in the European cluster [20]. The remaining outliers consisted of two South African samples and one Piaroa isolate. The Maorian and Amerindian sequences formed a subcluster with the highest branch support when increasing the stringency to a $75 \%$ bootstrap-value (M1 consensus analysis; see Methods). 


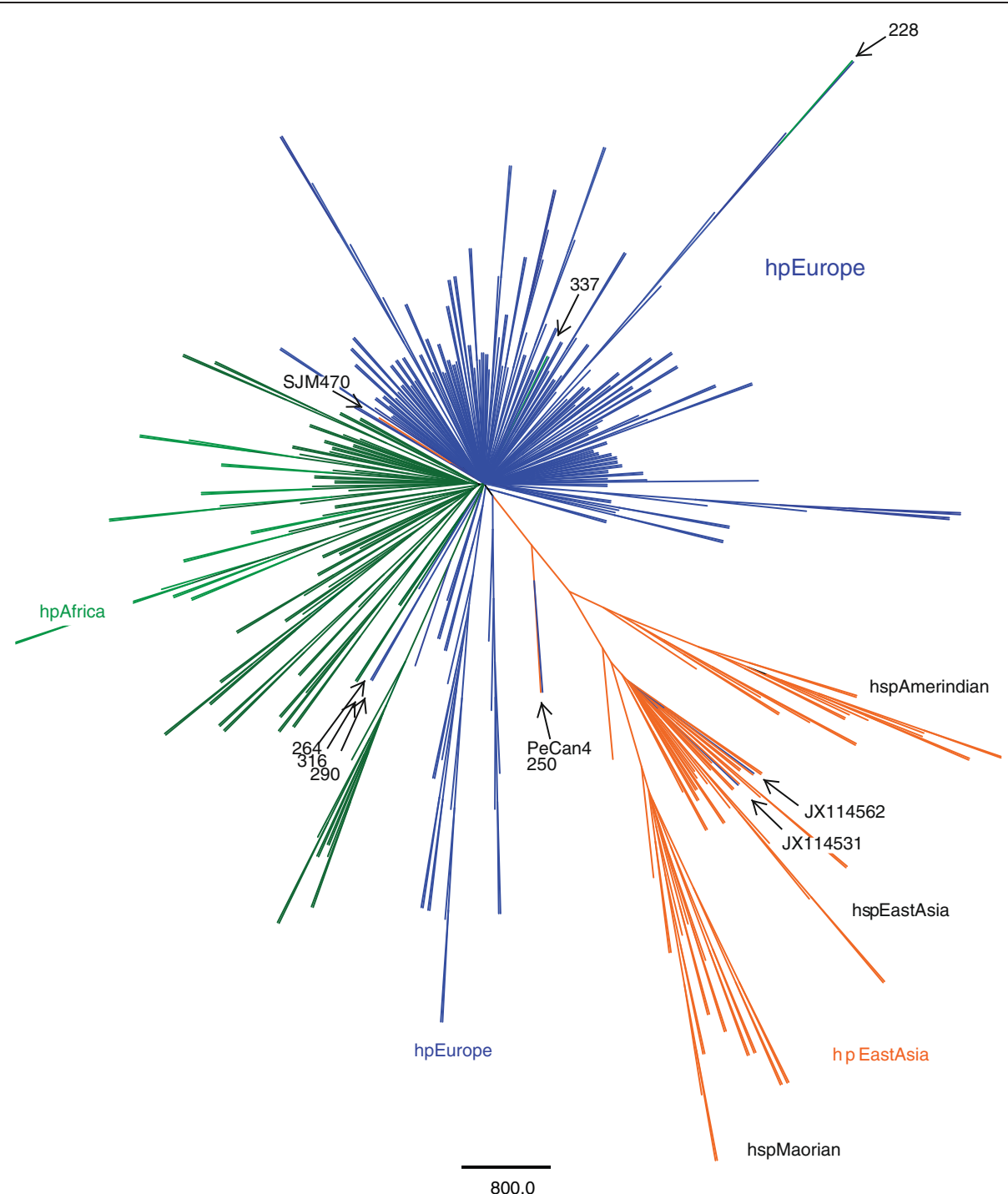

Figure 1 Phylogenetic tree of Helicobacter pylori housekeeping sequences. The seven concatenated HK genes were biogeographically classified: blue represents European strains (hpEurope), orange indicates the East Asian (hpEastAsia which includes the subpopulations hspAmerindian, hspEastAsian and hspMaorian) isolates, and green denotes African (hpAfrica) strains. The outliers are identified by black arrows (see Discussion for more information). Additional file 3: Table S1 contain label with corresponding MLST/GenBank ID. See Additional file 7: Figure S1 for complete labeling. This radial tree of 393 sequences is the majority rule consensus of 1000 maximum likelihood bootstrap replicates analyzed in PhyML with the GTR + I + G model and visualized in FigTree (see Methods for more details).

The phylogenetic tree based upon the pldA gene sequences is depicted in Figure 2 (see Additional file 1: Table S2 for annotations). The majority of the Korean sequences clustered in the same clade. This cluster contained two isolates sampled in Norway that had an East Asian cagA EPIYA-ABD genotype and came from patients of East Asian origin. The four Amerindian strains and five East Asian sequences from the 19 genome sequences were also found in this cluster. One of the samples isolated in Norway was from a patient of African origin and clustered with the four African sequences. The vacA genotype of this sample was $\mathrm{s} 1 \mathrm{~b}$, the genotype that is most common among the African, Spanish, and South American populations [21]. This pldA tree was unrooted and consisted of two main clusters, the East Asian cluster and the smaller African groups, nested within the vast majority of European sequences. The two African pldA sequences from the 199 and SouthAfrica7 genomes were found among the European sequences, as observed in the reference tree. Only three of the African strains formed a clade with $75 \%$ bootstrap analysis (in M1 consensus tree; data not shown).

The two pldA trees constructed using different models were compared in TOPD/FMTS using split distances. The average split distance was 0.58 , which indicated that 


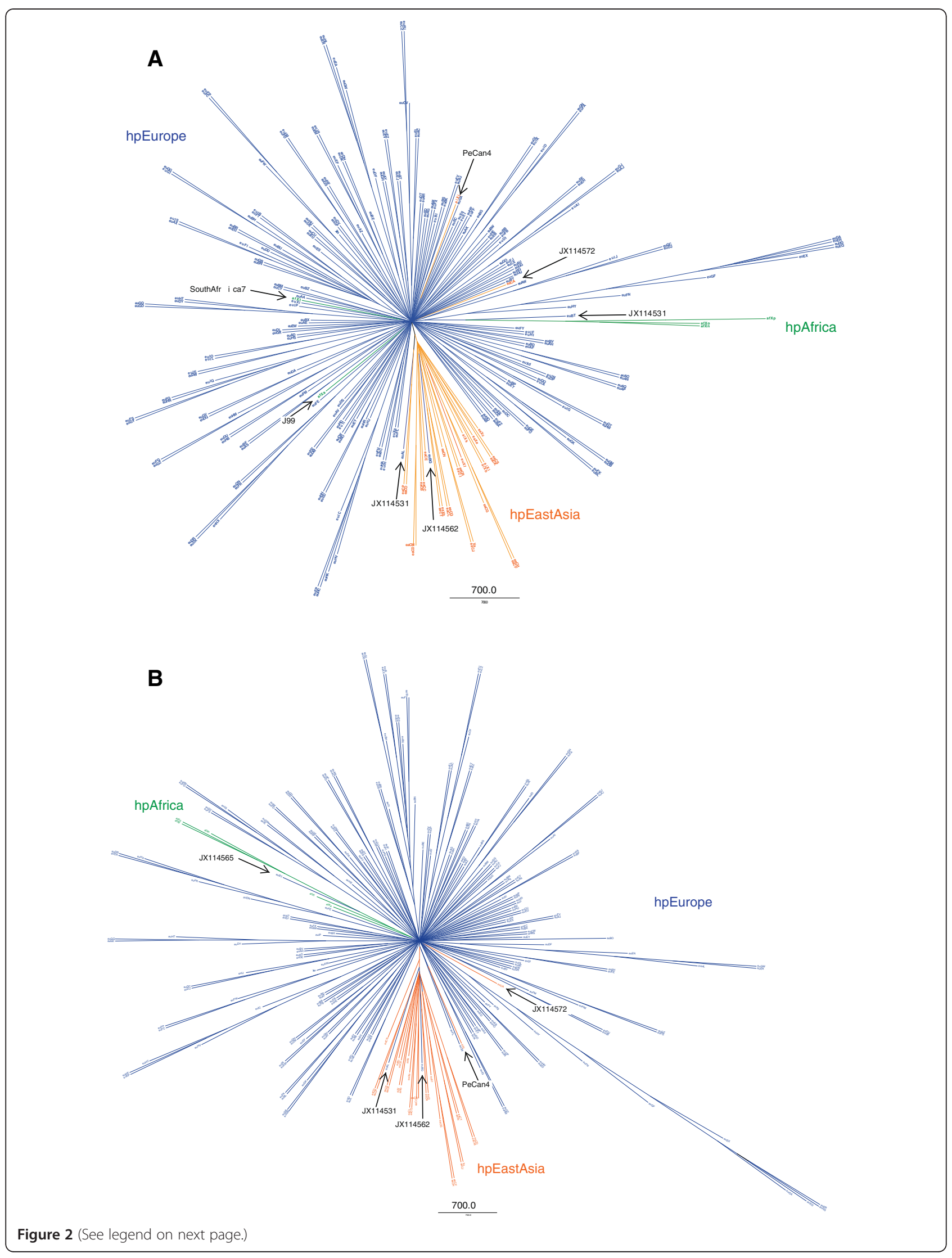


(See figure on previous page.)

Figure 2 Phylogenetic tree of Helicobacter pylori pldA sequences. The pldA sequences were biogeographically classified: blue represents European strains, orange indicates hpEastAsian isolates, and green denotes African strains (hpAfrica). The outliers are identified by black arrows (see Discussion for more information). Additional file 1: Table S2 contain label with corresponding GenBank Accession ID. Shown are radial consensus trees of 246 pldA sequences based on 1000 maximum likelihood bootstrap replicates analyzed in PhyML and visualized in FigTree (see Methods for details). Trees were constructed using either the $\mathrm{K} 80+\mathrm{G}+$ I model chosen by ModelTest (A) or the GTR + I $+\mathrm{G}$ model (B) as used to construct the reference tree (Figure 1).

the two trees were neither identical (split difference $=0$ ) nor completely different (1). A random split distance was calculated to analyze whether the split distances were significantly different. Because the random split distance resulted in a value close to $1(0.999885$, to be exact), our observations were probably not due to chance.

\section{Horizontal gene transfer analysis of pldA and OMPLA sequences}

The average GC content of the 19 pldA gene sequences was $40.18 \pm 0.35 \%$, while the average GC content of the corresponding 19 whole-genome sequences was $38.98 \pm$ $0.21 \%$, a significant difference $\left(P \approx 10^{-12}\right)$. The pldA mean GC content was greater than 1.5 standard deviations from the GC genomic mean, suggesting horizontal transfer. We further assessed whether the codon bias found in the pldA gene sequences could be due to biological or random effects. The codon adaptation index (CAI) was estimated by CAIcal [22] to be 0.77, while the eCAI estimate was 0.75 (with $\mathrm{p}<0.01$; $99 \%$ probability for $99 \%$ of the population). This yields a CAI/eCAI ratio of 1.03; a CAI value higher than the expected eCAI value indicates codon bias.

We collected 958 OMPLA sequences (listed in the Additional file 2: Table S3), of which 170 different species had pairwise sequence identities to $H$. pylori between $15 \%$ and $90 \%$. The vast majority of the protein sequences used in this study were from proteobacteria, with gamma proteobacteria accounting for nearly $72 \%$. In addition to proteobacteria, eight Bacteroidetes/Chlorobi (CFB) species were present. The average length of the OMPLA protein sequences was 320 amino acids (range 247-393), resulting in 79 residues in the final alignment. The phylogenetic tree of OMPLA is shown in Figure 3. The AtpA reference sequences had an average of 511 residues (range 499-548), and the final alignment contained 445 residues. The phylogenetic tree of AtpA is shown in Figure 4. Two Enterobacteriaceae species, Proteus vulgaris and Pantoea agglomerans (GammaPV and GammaPAa in Figure 3), see Additional file 3: Table S1 for the annotations used) were only found in the OMPLA dataset. The reference tree displays three distinct clusters of CFB, gamma, epsilon, and beta proteobacteria. However, the four delta sequences occurred in two separate clusters in both the reference and OMPLA trees. Two of them were sister to the epsilon sequences, as expected because they belong to the Epsilon/Delta subdivision within Proteobacteria. The main difference between the AtpA and OMPLA trees was that in the OMPLAtree the epsilon proteobacteria cluster was separated by multiple gamma clades. Helicobacter acinonychis and H. pylori were the two most distant sequences among all of the species in the OMPLA tree with a very strong bootstrap value (see Additional file 4). Sister to these two species were the remaining six Helicobacter spp., divided into two subclusters. The division of the epsilon group was also found using a $75 \%$ bootstrap support in the M1 consensus analysis) (see Additional file 5: Figure S2 and Additional file 6: Figure S3), indicating a strong branch that separates the Helicobacter sequences from the rest of the epsilon group. The largest cluster in the OMPLA phylogenetic tree consisted of about 50 gamma species. The remaining gamma sequences were found in closely-related subclusters. Some gamma proteobacteria were also related to either the epsilon, beta, or CFB subclusters.

\section{Adaptive molecular evolution in pldA sequences}

The SWAAP analysis resulted in an average $K_{a} / K_{s}$ ratio of $0.076 \pm 0.035$, indicating a protein under strong purifying selection. The codon-based $Z$-test bootstrap analysis confirmed that a vast majority $(98.86 \%)$ of the nucleotide sequences had a high probability $(p<0.01)$ of being under purifying selection.

Table 1 depicts the results of the test for positive selection in PAML. The two models that allowed positive selection, M2 and M8, fit our data better than the models, M1 and M7, that did not. The LRT showed that the M8 model best fit these data. This model estimated that fourteen sites $(4.63 \%)$ were under positive selection (Table 2), with $\omega=1.55$ and $85.83 \%$ were under purifying selection, with $\omega<0.2$. The M2 model estimated that $92.12 \%$ of the sites were under purifying selection, while $1.46 \%$ was positively selected. PAML estimated $\kappa \approx 4$ for M2 and M8.

\section{Discussion}

Brok et al. compared OMPLA protein orthologs from eleven different species and concluded that OMPLA contained 30 highly-conserved residues. The fact that OMPLA is present in a wide range of species, including H. pylori, and that the sequence is conserved across those species, 


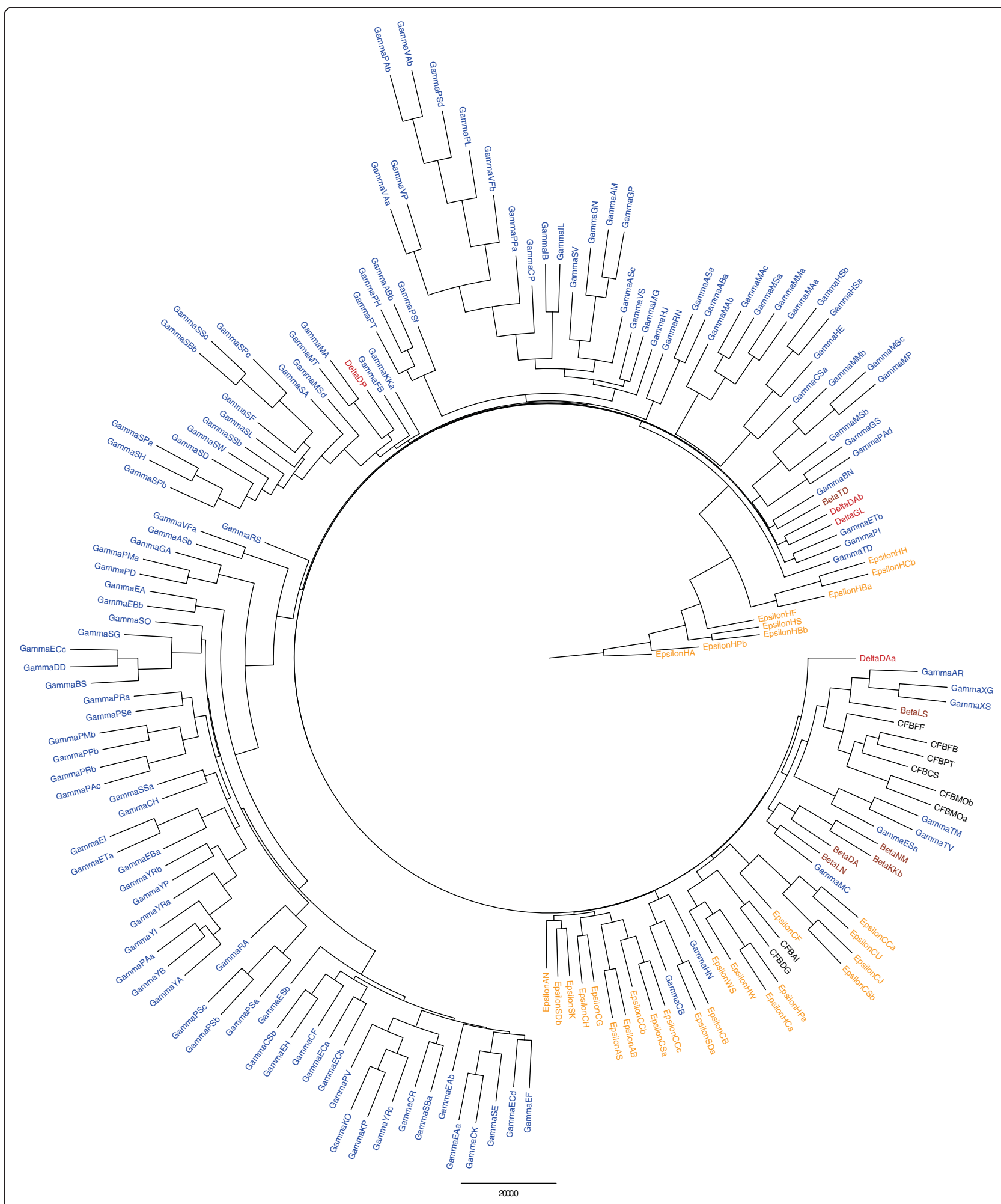

Figure 3 Phylogenetic tree of Proteobacteria OMPLA sequences. Majority-rule consensus tree of OMPLA sequences representing 171 species of gamma proteobacteria (blue), beta proteobacteria (brown), epsilon proteobacteria (orange), delta proteobacteria (red), and Bacteroidetes/Chlorobi (CFB; black). See Additional file 2: Table S3 for species labels used. 


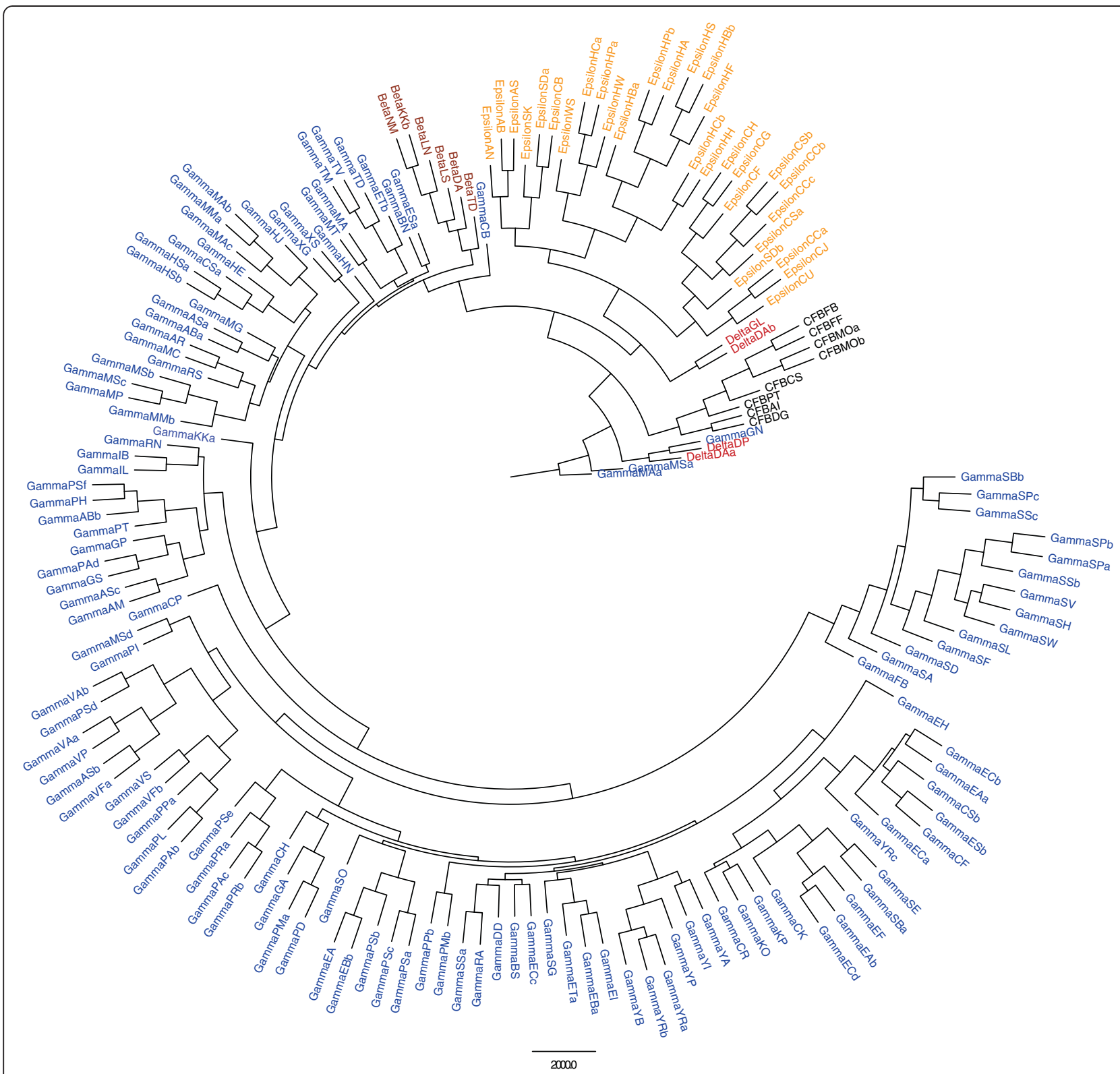

Figure 4 Phylogenetic tree of Proteobacteria AtpA sequences. Maximum likelihood majority-rule consensus tree of AtpA sequences derived from 169 species of gamma proteobacteria (blue), beta proteobacteria (brown), epsilon proteobacteria (orange), delta proteobacteria (red), and Bacteroidetes/Chlorobi (CFB; black). See Additional file 2: Table S3 for species labels used.

Table 1 Likelihood ratio test for model selection

\begin{tabular}{llll}
\hline Model & InL & LRT & $\mathbf{x}^{2}$ distribution \\
\hline M1 & -12515.96 & 47.04 & $>9$ with 2 d.o.f. $P<0.01$ \\
\hline M2 & -12492.44 & & \\
\hline M7 & -12521.64 & 83.94 & $>9$ with 2 d.o.f. $P<0.01$ \\
\cline { 1 - 2 } M8 & -12479.67 & & \\
\hline
\end{tabular}

Nested models with and without positive selection (M1 vs. M2 and M7 vs. M8) were compared in PAML. The $x^{2}$ distribution column shows the minimum likelihood ratio $(=2 \Delta \mathrm{lnL})$ necessary for the more complex of two models to be significantly better $(p<0.01)$. strongly indicates that its physiological role is significant [23]. This study aimed to better understand the significance of pldA, the gene coding for OMPLA, in H. pylori; an important gut bacterium in humans.

The $H$. pylori pldA gene had a low degree of variability and, thus, a conserved OMPLA protein sequence alignment. Housekeeping genes are essential for bacterial survival, and are thus highly conserved. The seven HK genes, atpA, efp, ppa, tphC, ureI, $\operatorname{trp} C$, and mutY, and the $p l d A$ gene are among the core genes that are found 
Table 2 Positively-selected sites in pldA of Helicobacter pylori

\begin{tabular}{llll}
\hline Site & Residue & Probability $\boldsymbol{\omega}>\mathbf{1}$ & Posterior probability \\
\hline 5 & W & $0.955^{*}$ & $1.48 \pm 0.20$ \\
\hline 6 & $\mathrm{~L}$ & $0.996^{* *}$ & $1.52 \pm 0.15$ \\
\hline 21 & $\mathrm{~S}$ & 0.830 & $1.37 \pm 0.33$ \\
\hline 27 & $\mathrm{I}$ & $1.000^{* *}$ & $1.52 \pm 0.14$ \\
\hline 34 & $\mathrm{R}$ & 0.576 & $1.15 \pm 0.42$ \\
\hline 40 & $\mathrm{I}$ & $0.999^{* *}$ & $1.52 \pm 0.14$ \\
\hline 50 & $\mathrm{~A}$ & $0.989^{*}$ & $1.51 \pm 0.15$ \\
\hline 59 & $\mathrm{P}$ & 0.858 & $1.39 \pm 0.29$ \\
\hline 137 & $\mathrm{D}$ & $1.000^{* *}$ & $1.52 \pm 0.14$ \\
\hline 144 & $\mathrm{D}$ & 0.760 & $1.32 \pm 0.33$ \\
\hline 153 & $\mathrm{M}$ & $1.000^{* *}$ & $1.52 \pm 0.14$ \\
\hline 209 & $\mathrm{P}$ & 0.851 & $1.39 \pm 0.31$ \\
\hline 211 & $\mathrm{G}$ & 0.836 & $1.38 \pm 0.30$ \\
\hline 278 & $\mathrm{~V}$ & $0.962^{*}$ & $1.48 \pm 0.15$
\end{tabular}

PAML predicted that 14 sites were under positive selection $(\omega>1)$ using Bayes empirical Bayes analysis for the M8 model. One asterisk $\left(^{*}\right)$ signifies a probability $>95 \%$ that $\omega>1$, while two asterisks $\left(^{* *}\right.$ ) signify a probability greater than $99 \%$. The best ancestral reconstruction is indicated by the highest value in the final posterior probability column.

in all H. pylori genomes sequenced to date [10]. The average sequence identity was higher in $p l d A$ and the molecular distance was lower than in the HK genes. The present study focused on analyzing pldA gene sequences that code for functional OMPLA proteins. In previous studies, we showed that most clinical isolates contain these coding pldAON sequences [13]. In this study, we included 155 isolates from a Norwegian population used in the Sørreisa study [24]. Most (97.5\%) of these isolates showed an $\mathrm{ON}$ phase variant, indicating that the gene encodes a functional OMPLA protein in most individuals. The homopolymeric tract induces a shift between a functional and a truncated protein by enabling a frameshift mutation. Wernegreen et al. postulated that selection will purge nucleotide changes that could interrupt the slippery tract, to maintain otherwise volatile sequences [25].

Why the pldA gene in H. pylori contains a homopolymeric tract is an enigma, and we explored whether its existence could be part of a gene deletion process or perhaps a mechanism needed to prevent activation in certain environments. The homopolymeric tract corresponded to residues $226-228$ in the translated OMPLA protein. Residue 278 was the most downstream site that was predicted to be under positive selection in this protein. The remaining twenty percent of the protein (after residue number 279) is under purifying selection, indicating functional constraints and implying that the protein is important to bacterial survival.

Genes under purifying selection are often involved in host-pathogen interactions. For example, purifying selection in orthopoxvirus is probably caused by host defense mechanisms [26]. However, pathogens must also evolve novel residues to evade the host immune system, resulting in positive selection on some residues [27]. Such positive selection has been shown in the flagellumcoding gene $f l A$, which is involved in adhesion in Aeromonas; nearly the entire protein was under purifying selection, while 17 residues were subject to positive selection [28]. Our analyses demonstrated purifying selection in most of the pldA sequence, while the remaining residues were predicted to be under positive selection. The positively-selected sites were scattered throughout the OMPLA protein. Petersen et al. concluded that positively-selected sites are exclusively located in the loops of outer membrane proteins [27]. In Rickettsiaceae, positively-selected sites were important for host-parasite interactions and were located at the exterior of the proteins [29]. The E. coli OMPLA structure had a beta-barrel transmembrane conformation [30]. Thus, one might reasonably assume that its positivelyselected sites are also within surface-exposed regions. The N-terminal end of the protein contained four positively-selected sites (two with $p \geq 99$ ), but they are most likely a signal sequence and not part of the mature protein.

Bacterial survival and persistence in the gastric mucosa requires adapting to an environment with constant fluctuating $\mathrm{pH}$. Helicobacter pylori adjusts to $\mathrm{pH}$ fluctuations in the stomach by regulating the expressions of nicheadapted genes such as urease and ferric uptake regulator (Fur), which protect against acidity by producing ammonium and modulating the expression of many genes under acidic conditions, respectively. Both genes are required for survival under acidic conditions. Fur mutants do not colonize well and are probably killed by environmental conditions in regions other than the final colonization sites, like in the mucus layer. The exact mechanism still remains unclear [31]. Because the pldA gene is required for growth at low $\mathrm{pH}$ [32] and active OMPLA protein is important for survival in acidic environments [33], the gene may be part of the acidic environment niche-adapted mechanism described. Helicobacter pylori OMPLA is an outer-membrane protein that is exposed to the continuously changing environment of the host, so its interactions should be optimized. This could cause some of the residues to be under positive selection pressure while the rest of the protein is conserved and is typically observed in proteins that are in the process of adapting to environmental changes [34].

Helicobacter pylori has demonstrated geographical clustering of its HK, virulence, and outer membrane protein genes in phylogenetic studies [11,12,35-38]. Because many genes with biogeographic patterns are highly conserved, we were interested in determining whether 
pldA gene sequences showed such partitioning. As a point of reference, we constructed a phylogenetic tree with the same sequences used by Falush et al. [11]. We found biogeographic patterns in both the reference HK and $p l d A$ gene trees; however, bootstrap values in both trees, indicates relatively weak support for the biogeographic clades perhaps due to the high sequence identity found in both alignments. The strongest clade found in the pldA tree (with $>75 \%$ bootstrap in the M1 consensus analysis; see Method section) contained three out of the four African H. pylori. However, one of the African isolates in the original analysis was not found in this clade. Thus, the African cluster could be due to the fact that the data were taken from same patient over many years [39].The HK reference tree contained sequences from around the world (using the Falush dataset and $H$. pylori genomes). The majority of the Amerindian samples clustered in the East Asian cluster, as reported for other genes [11,12,37]. However, although SJM180 is from a native American Peruvian isolate, it clustered with the European isolates, as described by Manjulata et al. [40]. The two samples in the East Asian subcluster were of East Asian origin and had an East Asian CagA genotype. The majority (86\%) of the East Asian pldA sequences contained two mutations (residues K168E and E176K). In future work, we would like to assess whether and how these two mutations influence OMPLA structure and function.

The phylogenetic trees were constructed to analyze the biogeography of the pldA sequences. In the process, we discovered that the best-fit substitution model for the pldA sequences was different from that of the HK genes. This indicates that the genes have not evolved under the same conditions and could be explained by HGT of the pldA gene. The K80 algorithm adjusts for transition to transversion (ts/tv) bias which was also confirmed with a high $\mathrm{ts} / \mathrm{tv}$ rate ratio $(\mathrm{\kappa} \sim 4)$ in the $p l d A$ dataset. We constructed two phylogenetic pldA trees, using the two models selected for reference and $p l d A$, to determine how the model would affect the geographical clustering. This would give insight into how pldA sequence evolution compares to that of the housekeeping genes. The HK reference genes represents the core genome diversity within $H$. pylori as they are scattered around the genome, flanked by conserved genes not expected to be under any immune selection [11].The two trees were found to be quite different, with a split distance ratio of 0.58 . Our findings were most likely due to biological effects rather than random bias. Interestingly, the only biogeographical difference observed between the two models was in the placement of the American $J 99$ isolate, which had African characteristics [11]. This sequence was found in the European cluster in the pldA K80 tree, while it clustered with the other
African sequences in both the HK and pldA GTR trees. These analyses could indicate that the genes have coevolved along different phylogenetic lines for a long time and that a possible HGT event involving pldA may have occurred relatively early in the evolution.

Our hypothesis of HGT was confirmed through both intra- and inter-species evolutionary analyses. Multiple analyses can infer HGT, including phylogenetic analysis of orthologs and estimates of codon bias and GC content. Our results indicated an ancient transfer, because the pldA tree had a similar biogeographical pattern to that of the reference tree. The OMPLA protein is mainly found in gamma proteobacteria. Horizontal gene transfer has been observed in, e.g., the CagPAI region, which has a lower GC content than the rest of the $H$. pylori genome [41]. The current study demonstrated a possible HGT event through the analysis of phylogeny, GC content, and codon bias. The GC content of the pldA sequences was slightly but significantly elevated compared to the rest of the $H$. pylori genome, and the difference was well above the accepted mean deviation threshold $[42,43]$. Although the $H$. pylori genomes as a whole lacked codon bias [44], further analysis was needed to ensure that the pldA gene was an exception to this conclusion. The CAI confirmed that the observed codon bias was most likely due to biological effects rather than artifact. Thus, the codon bias in the pldA gene suggested horizontal transfer [22]. Further confirmation for HGT was found in the phylogenetic analysis comparing OMPLA and AtpA sequences in which Helicobacter differed from the other epsilon proteobacteria. In particular, $H$. pylori and $H$. acinonychis were the two most divergent species in the OMPLA phylogenetic tree. These results were validated using an approximate likelihood ratio test in PhyML [45]. The phylogenetic tree of OMPLA conflicts with that of AtpA, indicating multiple HGT events. The species found outside of their expected clusters might have adapted quickly to environmental changes as a result of HGT events, which accelerate the rate of adaption [46]. This is illustrated in the epsilon cluster; three of the four non-epsilon bacteria in that clade colonize humans either as pathogenic bacteria or as part of the intestinal microbiota (see Figure 3 and Additional file 2: Table S3 for details).

\section{Conclusions}

The pldA gene in Helicobacter pylori has high nucleotide sequence identity due to purifying selection at the vast majority of residues. The result is a conserved $H$. pylori protein that likely has an evolutionarily stable function, although some probable interaction sites are subject to positive selection. Although HGT was detected by codon bias, GC content, and phylogenetic analysis, the 
biogeography of the pldA sequences indicated that the transfer was ancient. The protein structure of $H$. pylori OMPLA will yield a better understanding of the positively selected sites, which may be surface-exposed regions. Our analyses indicated that $p l d A$ may be a nicheadapted protein; it was horizontally acquired, is highly conserved, but positive selection occurs at sites needed for possible pathogenic interactions.

\section{Methods}

Helicobacter pylori sample collection and pldA sequencing The pldA gene of $227 \mathrm{H}$. pylori isolates was sequenced. The samples included 207 Norwegian and 20 Korean isolates. The Norwegian samples consisted of a total of 155 isolates from the Sørreisa study [24] and 52 isolates collected from four hospitals in the Oslo region. Among these isolates, 40 had been previously described [33]. The Oslo isolates included samples with known foreign origins; four isolates with Indo-European origins, two with Asian origins, and one with an African origin.

DNA was isolated using BioRobot M48 and MagAttract DNA Mini M48 Kit (Qiagen Inc., Valencia, CA, USA). The pldA gene, including short parts of the upand downstream genes, was amplified by polymerase chain reaction (PCR) with forward primer HP498/499-F (5'- ttatcgcgcctgtagtga -3') and reverse primer HP499/ 500-R (5'- tatgatcgctggcatgga - $\left.3^{\prime}\right)$ at an annealing temperature of $57^{\circ} \mathrm{C}$. The 1068 base pair (bp) pldA-gene was sequenced using the ABI BigDye Terminators v 1.1 Cycle Sequencing Kit (Applied Biosystems, Foster City, CA, USA) with the PCR primers and the internal sequencing primers HP498/499-R (5'-ggttgatattggggtggta3'), PLA-F (5'-tgtccaattcttggtatctc-3'), PLA-R (5' -atgcgataggtatagcctaag-3') and HP499/500-F (5'-tatgatcgctggcatgga-3'). The sequencing products were analyzed with an ABI PRISM 3130 Genetic Analyzer (Applied Biosystems) and the sequences were aligned using Sequencher software (Gene Codes Corporation, Ann Arbor, MI, USA). Finally, the DNA sequences were translated to complete protein sequences using RevTrans [47].

In addition to the 207 sequences collected in Norway that were included in this study, three additional isolates were sequenced and excluded because they coded for truncated proteins.

\section{CagA EPIYA genotyping}

To discriminate the East Asian from the European isolates, the CagA genotype was determined in the $20 \mathrm{Ko}-$ rean samples and 50 of the Norwegian ones. Amplification and sequencing of the 3' region of the $\operatorname{cagA}$ gene was performed as described by Yamaoka et al. [48].

\section{Amplification of vacA}

To confirm the African origin of one of the Norwegian samples, PCR amplification of the vacA signal sequence and mid-region was performed as described by Atherton et al. [49].

\section{Biogeographic analysis \\ Reference phylogenetic tree}

A reference phylogenetic tree was constructed using concatenated HK genes (atpA, efp, ppa, tphC, ureI, trpC, and $m u t Y$ ) collected from the $H$. pylori Multi Locus Sequence Typing (MLST) database [http://pubmlst.org/ helicobacter/] as described by Falush et al. [11]. In addition, 19 of the 29 currently-sequenced $H$. pylori genomes (See Appendix 1 for further annotation) collected from the National Center for Biotechnology Information (NCBI) database [http://www.ncbi.nlm.nih.gov] and four Norwegian isolates, sequenced according to the $H$. pylori MLST protocol, were used in the reference tree construction. In total, 393 sequences were aligned using ClustalW [50], and regions with gaps were removed using BioEdit [51]. Model selection in MEGA5 [52] was used to determine the best fit model for maximum likelihood (ML) analysis. PhyML v3.0 [53] was used to generate $1000 \mathrm{ML}$ bootstrap trees using the generalized time-reversible (GTR) model in which both the discrete gamma distribution $(+G)$ with five rate categories and invariable sites $(+\mathrm{I})$ were set to 0.61 , as this was the model with the lowest Bayesian Information Criterion score.

A consensus tree was constructed with Phylip's Consense package [54] and imported into FigTree v1.3.1 [http://tree.bio.ed.ac.uk/software/figtree/] for further visualization. These resolved trees contain monophyletic groups not contradicting more frequent groups with a $50 \%$ default threshold (majority-rule). As a supplement, a strict analysis with a higher threshold was included where only groups occurring more than $75 \%$ are included.

\section{PIdA phylogenetic tree}

The phylogenetic tree for pldA gene sequences was constructed using the same method as described for the reference tree. The pldA sequences were obtained through a Blast search of jhp_0451, limiting the search to $H$. pylori genome sequences. Only pldAON sequences coding for the entire OMPLA protein were included in this study. In addition, 19 of the 29 currently-sequenced $H$. pylori genomes collected from the NCBI database were aligned with the pldA gene sequences from the 227 isolates described in the current study. Genomes containing $p l d A$ genes that coded for truncated proteins were excluded from analyses. Reversed sequences from the genomes were converted to a 5'-3' reading frame 
using a reverse complement calculator [http://www.bioinformatics.org/sms/rev_comp.html]. The pldA alignment was stripped of gaps in BioEdit [51] and imported into MEGA5 [52] for model selection as described above. The alignments were analyzed in PhyML [53] using 1000 bootstraps and the Kimura two-parameter (K80) model with the gamma distribution (five rate categories) and invariant sites set to 0.34 and 0.53 , respectively; this model was found to be the best by MEGA5. A consensus tree was made in Phylip's Consense package [54] and represented as an unrooted radial tree in FigTree. The pldA dataset was also analyzed using the same model $(G T R+G+I)$ used for the reference tree. The two pldA trees generated using the GTR + G + I and $\mathrm{K} 80+\mathrm{G}+\mathrm{I}$ models were compared with the TOPD/FMTS software [55]. A random average split distance of 100 trees was also created to check if the differences observed were more likely to have been generated by chance.

\section{Comparison of pldA sequences with seven core housekeeping genes}

The average pairwise nucleotide identity for $p l d A$ and concatenated HK sequences was calculated in BioEdit [51]. The average genetic distance was calculated with the default K80 algorithm in MEGA5 [53,56].

\section{Horizontal gene transfer analysis of pldA and OMPLA sequences}

The DNA stability was determined by calculating the GC content of the pldA sequences using SWAAP 1.0.3 [57]. The GC content of the pldA sequences was compared to the overall GC content of the $H$. pylori genomes, and significant differences between these two groups were calculated using a two-tailed $t$-test (Excel 2003, Microsoft, Redmond, WA, USA). The Codon Adaptation Index (CAI) detects codon bias in a DNA sequence and indicates the possibility of HGT. CAIcal [22] was used to calculate the degree of codon bias and compare it to an estimated value from a reference set (eCAI).

The OMPLA protein sequences from 171 species were used for an intra-species phylogenetic analysis. Sequences were collected both from the KEGG database [58], using KEGG orthologs belonging to EC13.3.13, and, NCBI's similar sequence option. Both NCBI Batch Entrez [http:// www.ncbi.nlm.nih.gov/sites/batchentrez] and the Protein Information Resource (PIR) [59] were used to retrieve the protein sequences. Pairwise sequence identities were calculated for ClustalW aligned sequences in BioEdit [51]. Sequences with pairwise identities between $15-90 \%$ were kept, and the sequences (Appendix 1 lists all of the Protein IDs used) were re-aligned using the MAFFT web server [http://www.genome.jp/tools/mafft/], where the auto-option chose the FFT-NS-i model (an iterative method) [60]. Jalview [61] displayed the minimum, maximum, and average number of residues in the alignment. Poorly-aligned and divergent regions were removed using Gblocks [62]. PhyML [53] was used to construct a phylogenetic tree using the default variables (including LG ( Le and Gascuel) method estimates of the gamma and invariable sites). The same procedure was performed on the ATP synthase subunit alpha (AtpA) reference sequences that were collected for the species in the OMPLA protein list by searching the protein NCBI database (See Appendix 1 for the Protein IDs used). The consensus tree of AtpA and OMPLA sequences were generated from the 1000 PhyML bootstrap trees using Phylip's Consense package [54]. Results were visualized as circular trees using FigTree [http://tree.bio.ed.ac.uk/software/figtree/].

\section{Detection of adaptive molecular evolution of pldA sequences}

To study evolutionary divergence among the pldA sequences, the mean numbers of synonymous $\left(\mathrm{K}_{\mathrm{s}}\right)$ and nonsynonymous $\left(\mathrm{K}_{\mathrm{a}}\right)$ substitutions per site were estimated using the Nei and Gojobori method [63] in SWAAP [57]. The $K_{s}$ value is the mean number of synonymous (silent) substitutions per site, while $K_{a}$ represents the mean number of nonsynonymous substitutions per site (a change of amino acid is observed). The MEGA5 [52] codon-based Z-test for purifying selection was used to estimate the probability of rejecting strict neutrality (null hypothesis where $K_{a}$ equals $K_{s}$ ) in favor of the alternate hypothesis $\mathrm{K}_{\mathrm{a}}<\mathrm{K}_{\mathrm{s}}$.

The PAML program [64] estimates the nonsynonymous/synonymous ratio, omega $(\omega)$, using maximum likelihood codon substitution models. In this study, four different models (M1, M2, M7, and M8) were used to estimate $\omega$ as described by Yang et al. [65]. These models are nested pairs in which one (M1 and M7) does not allow for positive selection, while the other (M2 and M8) includes an additional parameter to detect positively selected sites. The neutral model M1 assumes two classes of proteins, highly conserved codons $(\omega=0)$ and neutral codons $(\omega=1)$, and is nested within the M2 model, which has a third category for positive selection $(\omega>1)$. The two most advanced models, M7 and M8, use a discrete $B$ distribution; M8 has an extra class of codons that allows positive detection $(\omega>1)$ and simplifies to M7. The two pairs of nested models (M1 vs. M2 and M7 vs. M8) were compared using the likelihood ratio test (LRT) statistic, where $2 \Delta \operatorname{lnL}$ equals $2 *\left(\ln L_{1}-\right.$ $\ln L_{0)}$. The $\ln L_{1}$-value is the log-likelihood for the more advanced model and $\ln \mathrm{L}_{0}$ is the log-likelihood for the simpler model. The $2 \Delta \ln L$ value follows a $X^{2}$ distribution, where the degree of freedom is the difference in the number of parameters used in the two models. The 
identification of positive selected sites implemented in PAML uses Bayes empirical Bayes where the posterior probabilities of each codon was calculated from the site class of the M2 and M8 models; sampling errors have been accounted for through Bayesian prior [66,67]. A pldA tree generated in PhyML using the K80 model (the best fit as determined in MEGA5) was used in the PAML analysis. PAML also calculated possible transition (ts) to transversion (tv) bias ( $\mathrm{k}=\mathrm{ts} / \mathrm{tv})$.

\section{Nucleotide sequence accession numbers}

The nucleotide sequences analyzed in this study were deposited in the NCBI GenBank database under accession numbers JX114520 to JX114746.

\section{Appendix 1: Protein and gene annotation IDs}

The 19 genomes used, and their pldA EMBL IDs, along with their expected Helicobacter pylori biogeographic traits are listed below:

- European traits: HPAG1, Lithuania75, P12, 52, 26695, SJM180, India7 [NCBI NC_008086.1, CP002334.1, NC_011498.1, CP001680.1, NC_000915.1, NC_014560.1, CP002331.1];

- African traits: J99, 2017, 2018, 908 and SouthAfrica7 [NCBI NC_000921.1, CP002571.1, CP002572.1, CP002184.1, CP002336.1, CP002337.1, ];East Asian traits: F16, F30, 35A, PeCan4, Shi470, 83 and Sat464 [NCBI AP011940.1, AP011941.1, CP002096.1, CP002074.1, NC_010698.2, CP002605.1, CP002071.1].

Genes that coded for truncated proteins (pldA OFF) were not included in this study.

\section{The 169 AtpA sequences used in the HGT analysis}

AtpA [NCBI: EHB93466.1, EEB65020.1, EGK01617.1, EAZ96951.1, EIA10014.1, EHO10730.1, EHQ42656.1, EAS72787.1, AAZ48838.1, ACV28038.1, EGK08739.1, EEG10159.1, EDM84731.1, EGC64000.1, AAZ98752.1, ACN14443.1, EAT15601.1, ADW17434.1, ACD96878.1, EFU68802.1, ADG93995.1, BAK73949.1, EDZ61621.1, EIB16597.1, EAT97454.1, EAU01020.1, ABK81906.1, EEV18591.1, ABS52242.1, ADN90332.1, EET80348.1, EHL90702.1, EFU71262.1, CAJ99396.1, EEO22948.1, CCF80240.1, EFR48376.1, EFR47618.1, CBY83548.1, AAP77024.1, EEQ62944.1, AAD08176.1, EFX42435.1, EEO26643.1, ABB44682.1, ACZ11550.1, ADR33423.1, CAE09651.1, CAL18176.1, EAW26695.1, AEB00215.1, EEY85631.1, EDX91133.1, CAQ80745.1, AEF05917.1, EAR22945.1, EHD23759.1, AAO91433.1, EHL85304.1, ACQ68874.1, YP_001451687.1, AAZ26667.1, CBG90709.1, ABE60630.1, ABU79194.1, ADN00765.1, CBJ48151.1, AEN67142.1, EDS93360.1, EFV38590.1, CAX62120.1,
EFC54899.1, AEW75952.1, CAG77409.1, CAP78192.1, CAQ91467.1, GAB51972.1, ACR71021.1, EHQ52780.1, ABP62783.1, EFE21167.1, EGW54096.1, ADN77981.1, AEC17221.1, AEP31454.1, GAB56517.1, AEE25184.1, CBV44330.1, ABC33685.1, ACX97137.1, EHK61102.1, EGP19691.1, EAQ31531.1, AAV83453.1, EHS93248.1, AEK00623.1, EGL54277.1, ADP99760.1, EDM48519.1, ABM20945.1, EGE27602.1, EAW32658.1, EHJ04715.1, ADZ93414.1, AEF56544.1, EBA00697.1, EAQ64801.1, ABR73359.1, EDM65164.1, EEF79996.1, EAS66680.1, EEB44391.1, ABG42796.1, EEX50537.1, EGI73341.1, ABM05406.1, GAA05763.1, AET16617.1, EEI49869.1, EAS45491.1, EEG87182.1, EFE51392.1, EFB70640.1, EFM18673.1, ADU71268.1, EIB97664.1, EAR55051.1, EDU61485.1, GAA64110.1, EAR27048.1, AEX54272.1, GAB59628.1, EAR11223.1, ABM01849.1, CCC32467.1, AEG13513.1, ABE57027.1, CAR35257.1, ABI73872.1, BAE75687.1, ABZ78836.1, ABO25710.1, EFA14838.1, ABV89552.1, ACJ31773.1, ADV56630.1, EIC83933.1, ABV39090.1, EGM67869.1, BAJ04308.1, ACA89149.1, EGV28007.1, EGV18064.1, EGZ46719.1, EAS75526.1, EAS62862.1, AAW87061.1, EEX40605.1, EGF42098.1, EDL54805.1, EGD19228.1, ZP_09853641.1, EEP94770.1, EEQ08006.1, EEQ18999.1, YP_654074.1, EEQ03775.1, EEQ00089.1, EHM50189.1].

\section{The 171 OMPLA sequences used in the HGT analysis}

OMPLA: [NCBI EAZ99640.1, ADW17991.1, EHQ52957.1, EGL54504.1, EGK10785.1, EGV19191.1, CAQ79680.1, EEY87557.1, EEB64935.1, EHO08344.1, EGC65261.1, EIA07918.1， EAR22975.1， EGD17737.1， EHK60019.1, AAZ46833.1, AAZ96049.1, EGP20312.1, EHB92999.1, EDM47887.1, ZP_09857083.1, EHJ06187.1, EAS71795.1, EDM84432.1, ABM17560.1, GAB54415.1, AEP29176.1, EGK01773.1， CAL17552.1， EEF79803.1， ACN14146.1, ADR35309.1, EDX88885.1, EHQ44562.1, EET80219.1, ABB43297.1, AEF53991.1, ADP95974.1, AEE23125.1, ADZ90582.1, EAR10180.1, EAQ32639.1, CBV41928.1, EDL54875.1, ABR72196.1, EAQ63108.1, ACV26008.1, EAS65010.1, EGZ42951.1, EGV31023.1, ZP_01234806.1, GAA04467.1, EEG09398.1, EDZ63591.1, EAR56640.1, EGF41493.1, AAV83321.1, AEF05108.1, AEA97203.1, EAU01382.1, ACQ67963.1, CAD32066.1, EAS76085.1, ADG93813.1, ABM05176.1, EAZ96211.1, ABE58799.1, ABS52347.1, AAW86051.1, ABG40599.1, EDM67950.1, EEV17429.1, ADN76662.1, EHD19745.1, ABC27991.1, ADN00421.1, EFB72463.1, BAK72959.1, ABV35292.1, BAJ03481.1, GAB60703.1, ACA85081.1, EAR28662.1, EGI74195.1, EEB46686.1, GAA62323.1, EAT16431.1, EAS40470.1, ACJ30728.1, ACD97136.1, AEN66963.1, EAW30307.1, ABZ78078.1, EFE52140.1, EDU58126.1, EFC53577.1, ABO22543.1, ABV11329.1, ACX96270.1, EAW29496.1, EIC83527.1, ABV85988.1, ABM01096.1, BAE75613.1， CAR35328.1， EEP97888.1， EGM70992.1, 
CAA54224.1, EFA15011.1, ABU78936.1, AET16551.1, EFU69622.1, ABI73025.1, EGW55053.1, ACZ13275.1, EEQ18686.1, EEP94174.1, ABE54243.1, AEG10235.1, CAQ91143.1, EHL84474.1, CAX57751.1, 1FW2, ABP62630.1, EHM51878.1, GAB53576.1, EHS92439.1, CBG90636.1, EFV38511.1, EAT97941.1, CCC32538.1, CAA54223.1, EIB97812.1, EEG87253.1, CAE01133.1, ADV55550.1, EDS90253.1, EEX50977.1, EEQ03301.1, AAD03498.1, AEX54094.1, ABK82197.1, ACR67376.1, EEQ04956.1, EFM18818.1, EEI47649.1, ADU67494.1, ACV41773.1, CAA71915.1, EFE21458.1, AEC17546.1, CAE09192.1, CAJ99604.1, EEO25025.1, CCF79664.1, EES88872.1, EFR45804.1, CBY82368.1, AAP77450.1, EEQ63232.1, AAD07564.1, EFX41646.1, EEO25572.1] [SwissProt C9PFN8_VIBFU, D4ICT7_ERWAE, D6DP51_ENTCL, E6LA24_CAMUP, Q0P8Q8_CAMJE, Q83E43_COXBU] [PRF 3020410HLP, 3117429CWR].

\section{Additional files}

\section{Additional file 1: Table S2. pldA labeling. Lists the NCBI accession number with the corresponding labelling used in Figure $2 a$ and $b$.}

Additional file 2: Table S3. Proteobacteria labelling. This table contains the abbreviated Proteobacteria names found in Figures 3 and 4 with the corresponding full bacteria name.

Additional file 3: Table S1. Housekeeping labelling. This table lists the MLST ID or NCBI accession number of the 7 concatenated housekeeping genes used in the analysis depicted in Figure 1.

Additional file 4: Extended majority rule consensus tree (outfiles). The outfiles that are the CONSENSE software results file from the phylogenetic trees from the phylogenetic analysis of housekeeping (Figure 1), pldA (Figure 2a and b), OMPLA (Figure 3) and AtpA (Figure 4).

Additional file 5: Figure S2. Phylogenetic tree of Proteobacteria OMPLA sequences. Additional file 5 is a strict analysis of the OMPLA sequences found Figure 3. In this analysis, a higher threshold is used where only groups occurring more than $75 \%$ is included (M75).

Additional file 6: Figure S3. Phylogenetic tree of Proteobacteria AtpA sequences. Additional file 5 is a strict analysis (M75) of the OMPLA sequences found Figure 4.

Additional file 7: Figure S1. Phylogenetic tree of H. pylori housekeeping sequences. Additional file 7 supplements Figure 1 with complete labelling.

\footnotetext{
Abbreviations

AtpA, ATPase F1 subunit alpha; Bp, Base pairs; CagPAl, Cag pathogenicity island; CAl, Codon adaptation index; CFB, Cytophaga-flavibacteriumbacteroides; eCAl, Estimated codon adaptation index; FUR, Ferric uptake regulator; GTR, Generalized time-reversible algorithm; HGT, Horizontal gene transfer; HK, Housekeeping genes; M2-M8, PAML models 2-8; LG, Le and gascuel substation matrix (2008); LRT, Likelihood ratio test; NCBI, National Center for Biotechnology Information; OMPLA, Outer membrane phospholipase A; PCR, Polymerase chain reaction; PAML, Phylogenetic analysis of maximum likelihood; ML, Maximum likelihood; MLST, Multi locus sequence typing; K80, Kimura-2-parameter algorithm; Ka, Synonymous-value (Dn); Ks, Nonsynonymous-value (Ds); PCR, Polymerase chain reaction; PIR, Protein information resource; T4SS, Type IV secretion system; $\mathrm{Ts} / \mathrm{TV}$, Transition /transversion ( $\mathrm{k}$ ).
}

\section{Competing interests}

The authors declare no competing interests.

\section{Authors' contributions}

HSV planned the study design and performed all the bioinformatic analyses YY made the Korean isolates available for this study and provided insightful comments with regard to outer membrane proteins of $H$. pylori. TT sequenced pldA, genotyped CagA from the Norwegian and Korean isolates and contributed throughout the process. GB supervised the study. All authors read and approved the final manuscript.

\section{Acknowledgements}

This research was supported by grants from the Norwegian South-Eastern Regional Health Authority. We thank Professor Gert Vriend, Radboud University, Nijmegen, for critically reading this manuscript. We also thank University of Oslo Bioportal and CMBI, Njimegen University, for providing resources to support our analyses.

\section{Author details}

'Department of Clinical Molecular Biology and Laboratory Sciences (EpiGen), Division of Medicine, Akershus University Hospital, University of Oslo, Oslo, Norway. ${ }^{2}$ Department of Medicine-Gastroenterology, Michael E. DeBakey Veterans Affairs Medical Center and Baylor College of Medicine, Houston, TX, USA. ${ }^{3}$ Institute of Health and Society, University of Oslo, Oslo, Norway.

${ }^{4}$ Centre for Laboratory Medicine, Østfold Hospital Trust, Fredrikstad, Norway.

Received: 13 February 2012 Accepted: 31 August 2012

Published: 13 September 2012

\section{References}

1. Yoshiyama $H$, Nakazawa T: Unique mechanism of Helicobacter pylori for colonizing the gastric mucus. Microbes Infect 2000, 2(1):55-60.

2. Bergman M, del Prete G, van Kooyk Y, Appelmelk B: Helicobacter pylori phase variation, immune modulation and gastric autoimmunity. Nat Rev Microbiol 2006, 4(2):151-159.

3. Sipponen P, Hyvärinen H, Seppälä K, Blaser M: Review article: pathogenesis of the transformation from gastritis to malignancy. Aliment Pharmacol Ther 1998, 12(Suppl 1):61-71.

4. Israel D, Peek RJ: The role of persistence in Helicobacter pylori pathogenesis. Curr Opin Gastroenterol 2006, 22(1):3-7.

5. Kusters J, van Vliet A, Kuipers E: Pathogenesis of Helicobacter pylori infection. Clin Microbiol Rev 2006, 19(3):449-449.

6. Covacci A, Rappuoli R: Helicobacter pylori: molecular evolution of a bacterial quasi-species. Curr Opin Microbiol 1998, 1(1):96-102.

7. Kuipers E, Israel D, Kusters J, Gerrits M, Weel J, van Der Ende A, van Der Hulst R, Wirth H, Höök-Nikanne J, Thompson S, et al: Quasispecies development of Helicobacter pylori observed in paired isolates obtained years apart from the same host. J Infect Dis 2000, 181(1):273-282.

8. Nedenskov-Sørensen P, Bukholm G, Bøvre K: Natural competence for genetic transformation in Campylobacter pylori. J Infect Dis 1990, 161(2):365-366.

9. Smeets L, Kusters J: Natural transformation in Helicobacter pylori: DNA transport in an unexpected way. Trends Microbiol 2002, 10(4):159-162.

10. McClain MS, Shaffer CL, Israel DA, Peek RMJ, Cover TL: Genome sequence analysis of Helicobacter pylori strains associated with gastric ulceration and gastric cancer. BMC Genomics 2009, 10:3.

11. Falush D, Wirth T, Linz B, Pritchard J, Stephens M, Kidd M, Blaser M, Graham $D$, Vacher S, Perez-Perez G, et al: Traces of human migrations in Helicobacter pylori populations. Science 2003, 299(5612):1582-1585.

12. Linz B, Balloux F, Moodley Y, Manica A, Liu H, Roumagnac P, Falush D, Stamer C, Prugnolle F, van der Merwe S, et al: An African origin for the intimate association between humans and Helicobacter pylori. Nature 2007, 445(7130):915-918.

13. Yamaoka Y, Kato M, Asaka M: Geographic differences in gastric cancer incidence can be explained by differences between Helicobacter pylori strains. Intern Med 2008, 47(12):1077-1083

14. Zhong Q, Shao S, Cui L, Mu R, Ju X, Dong S: Type IV secretion system in Helicobacter pylori: a new insight into pathogenicity. Chin Med J (Engl) 2007, 120(23):2138-2142.

15. Olbermann P, Josenhans $C$, Moodley $Y$, Uhr M, Stamer C, Vauterin M, Suerbaum S, Achtman M, Linz B: A global overview of the genetic and functional diversity in the Helicobacter pylori cag pathogenicity island. PLoS Genet 2010, 6(8):e1001069. 
16. Dorrell N, Martino M, Stabler R, Ward S, Zhang Z, McColm A, Farthing M, Wren B: Characterization of Helicobacter pylori PIdA, a phospholipase with a role in colonization of the gastric mucosa. Gastroenterology 1999, 117(5):1098-1104

17. Ziprin R, Young C, Byrd J, Stanker L, Hume M, Gray S, Kim B, Konkel M: Role of Campylobacter jejuni potential virulence genes in cecal colonization. Avian Dis 2001, 45(3):549-557

18. Tannaes T, Bukholm I, Bukholm G: High relative content of lysophospholipids of Helicobacter pylori mediates increased risk for ulcer disease. FEMS Immunol Med Microbiol 2005, 44(1):17-23.

19. Kawai M, Furuta Y, Yahara K, Tsuru T, Oshima K, Handa N, Takahashi N, Yoshida M, Azuma T, Hattori M, et al: Evolution in an oncogenic bacterial species with extreme genome plasticity: Helicobacter pylori East Asian genomes. BMC Microbiol 2011, 16(11):104.

20. de Sablet T, Piazuelo M, Shaffer C, Schneider B, Asim M, Chaturvedi R, LE B, Sicinschi L, Delgado A, Mera R, et al: Phylogeographic origin of Helicobacter pylori is a determinant of gastric cancer risk. Gut 2011, 60(9):1189-1195

21. Nagiyev T, Yula E, Abayli B, Koksal F: Prevalence and genotypes of Helicobacter pylori in gastric biopsy specimens from patients with gastroduodenal pathologies in the Cukurova region of Turkey. I Clin Microbiol 2009, 47(12):4150-4153.

22. Puigbò P, Bravo I, Garcia-Vallve S: CAlcal: a combined set of tools to assess codon usage adaptation. Biol Direct 2008, 3:38.

23. Brok R, Boots A, Dekker N, Verheij H, Tommassen J: Sequence comparison of outer membrane phospholipases A: implications for structure and for the catalytic mechanism. Res Microbiol 1998, 149(10):703-710.

24. Bernersen B, Johnsen R, Bostad L, Straume B, Sommer A, Burhol P: Is Helicobacter pylori the cause of dyspepsia? BMJ 1992, 304(6837):1276-1279.

25. Wernegreen J, Kauppinen S, Degnan P: Slip into something more functional: selection maintains ancient frameshifts in homopolymeric sequences. Mol Biol Evol 2010, 27(4):833-839.

26. Esteban D, Hutchinson A: Genes in the terminal regions of orthopoxvirus genomes experience adaptive molecular evolution. BMC Genomics 2011, $12: 261$.

27. Petersen L, Bollback J, Dimmic M, Hubisz M, Nielsen R: Genes under positive selection in Escherichia coli. Genome Res 2007. 17(9):1336-1343.

28. Farfán M, Miñana-Galbis D, Fusté M, Lorén JG: Divergent evolution and purifying selection of the flaA gene sequences in Aeromonas. Biol Direct 2009, 4:23.

29. Jiggins F, Hurst G, Yang Z: Host-symbiont conflicts: positive selection on an outer membrane protein of parasitic but not mutualistic Rickettsiaceae. Mol Biol Evol 2002, 19(8):1341-1349.

30. Snijder H, Ubarretxena-Belandia I, Blaauw M, Kalk K, Verheij H, Egmond M, Dekker N, Dijkstra B: Structural evidence for dimerization-regulated activation of an integral membrane phospholipase. Nature 1999 401(6754):717-721.

31. Gancz H, Censini S, Merrell D: Iron and pH homeostasis intersect at the level of Fur regulation in the gastric pathogen Helicobacter pylori. Infect Immun. Infect Immun 2006, 74(1):602-614.

32. Reid A, Pandey R, Palyada K, Whitworth L: E D, Stintzi A: Identification of Campylobacter jejuni genes contributing to acid adaptation by transcriptional profiling and genome-wide mutagenesis. App/ Environ Microbiol 2008, 74(5):1598-1612

33. Tannaes T, Dekker N, Bukholm G, Bijlsma J, Appelmelk B: Phase variation in the Helicobacter pylori phospholipase $A$ gene and its role in acid adaptation. Infect Immun 2001, 69(12):7334-7340.

34. Padhi A, Verghese B, Otta S: Detecting the form of selection in the outer membrane protein $C$ of Enterobacter aerogenes strains and Salmonella species. Microbiol Res 2009, 164(3):282-289.

35. Oleastro M, Cordeiro R, Ménard A, Yamaoka Y, Queiroz D, Mégraud F, Monteiro L: Allelic diversity and phylogeny of homB, a novel co-virulence marker of Helicobacter pylori. BMC Microbiol 2009, 9:248.

36. Pride D, Blaser M: Concerted evolution between duplicated genetic elements in Helicobacter pylori. J Mol Biol 2002, 316(3):629-642.

37. Cao P, Lee K, Blaser M, Cover T: Analysis of hopQ alleles in East Asian and Western strains of Helicobacter pylori. FEMS Microbiol Lett 2005, 251(1):37-43.
38. Yamaoka Y, Orito E, Mizokami M, Gutierrez O, Saitou N, Kodama T, Osato M, Kim J, Ramirez F, Mahachai V, et al: Helicobacter pylori in North and South America before Columbus. FEBS Lett 2002, 517(1-3):180-184.

39. Avasthi T, Devi S, Taylor T, Kumar N, Baddam R, Kondo S, Suzuki Y, Lamouliatte H, Mégraud F, Ahmed N: Genomes of two chronological isolates (Helicobacter pylori 2017 and 2018) of the West African Helicobacter pylori strain 908 obtained from a single patient. J Bacteriol 2011, 193(13):3385-3386.

40. Devi S, Ahmed I, Khan A, Rahman S, Alvi A, Sechi L, Ahmed N: Genomes of Helicobacter pylori from native Peruvians suggest admixture of ancestral and modern lineages and reveal a western type cag-pathogenicity island. BMC Genomics 2006, 27(7):191.

41. Salaün L, Saunders N: Population-associated differences between the phase variable LPS biosynthetic genes of Helicobacter pylori. BMC Microbiol 2006, 6:79.

42. Penn K, Jenkins C, Nett M, Udwary D, Gontang E, McGlinchey R, Foster B, Lapidus A, Podell S, Allen E, et al: Genomic islands link secondary metabolism to functional adaptation in marine Actinobacteria. ISME J 2009, 3(10):1193-1203.

43. Raiford D, Krane D, Doom T, Raymer M: Automated isolation of translational efficiency bias that resists the confounding effect of GC (AT)-content. IEEE/ACM Trans Comput Biol Bioinform 2010, 7(2):238

44. Lafay B, Atherton J, Sharp P: Absence of translationally selected synonymous codon usage bias in Helicobacter pylori. Microbiology 2000, 146(Pt 4):851-860

45. Anisimova M, Gascuel O: Approximate likelihood ratio test for branchs: a fast, accurate and powerful alternative. Syst Biol 2006, 55(4):539-552.

46. Haggerty L, Martin F, Fitzpatrick D, Mclnerney J: Gene and genome trees conflict at many levels. Phil Trans R Soc B 2009, 364(1527):2209-2219.

47. Wernersson R, Pedersen A: RevTrans: multiple alignment of coding DNA from aligned amino acid sequences. Nucleic Acids Res 2003, 31(13):3537-3539.

48. Yamaoka Y, Kodama T, Kashima K, Graham D, Sepulveda A: Variants of the 3' Region of the cagA gene in Helicobacter pylori Isolates from patients with different H. pylori-associated diseases. J Clin Microbiol 1998, 36(8):2258-2263.

49. Atherton J, Cao P, Peek RJ, Tummuru M, Blaser M, Cover T: Mosaicism in vacuolating cytotoxin alleles of Helicobacter pylori. Association of specific vacA types with cytotoxin production and peptic ulceration. J Biol Chem 1995, 270(30):17771-17777.

50. Larkin M, Blackshields G, Brown N, Chenna RMP, McWilliam H, Valentin F, Wallace IM, Wilm A, Lopez R, Thompson JD, Gibson TJ, Higgins DG: ClustalW and ClustalX version 2. Bioinformatics 2007, 23(21):2947-2948.

51. Hall T: BioEdit: a user-friendly biological sequence alignment editor and analysis program for Windows 95/98/NT. Nucl Acids Symp Ser 1999, 41:95-98.

52. Tamura K, Peterson D, Peterson N, Stecher G, Nei M, Kumar S: MEGA5: Molecular Evolutionary Genetics Analysis using Maximum Likelihood, Evolutionary Distance, and Maximum Parsimony Methods. Mol Biol Evol 2011, 28:2731-2739.

53. Guindon S, Dufayard J, Lefort V, Anisimova M, Hordijk W, Gascuel O: New algorithms and methods to estimate maximum-likelihood phylogenies: assessing the performance of PhyML 3.0. Syst Biol 2010, 59(3):307-321.

54. Felsenstein J: PHYLIP (Phylogeny Inference Package) version 3.6. In Distributed by the author. Seattle: Department of Genome Sciences, University of Washington; 2004

55. Puigbo P, Garcia-Vallve S, Mclnerney J: TOPD/FMTS: a new software to compare phylogenetic trees. Bioinformatics 2007, 23:1556-1558.

56. Kimura $\mathrm{M}$ : A simple method for estimating evolutionary rates of base substitutions through comparative studies of nucleotide sequences. J Mol Evol 1980, 16(2):111-120.

57. Pride D: SWAAP Version 1.0 -sliding windows alignment analysis program: a tool for analyzing patterns of substitutions and similarity in multiple alignments. In Distributed by the author. 2000.

58. Ogata H, Goto S, Sato K, Fujibuchi W, Bono H, Kanehisa M: KEGG: Kyoto Encyclopedia of Genes and Genomes. Nucleic Acids Res 1999, 27(1):29-34

59. Wu CH, Huang $H$, Arminski L, Castro-Alvear J, Chen $Y$, Hu ZZ, Ledley RS, Lewis KC, Mewes HW, Orcutt BC, et al: The Protein Information Resource: an integrated public resource of functional annotation of proteins. Nucleic acids research 2002, 30(1):35-37. 
60. Katoh K, Toh H: Recent developments in the MAFFT multiple sequence alignment program. Brief Bioinform 2008, 9(4):286-298.

61. Waterhouse A, Procter J, Martin D, Clamp M, Barton G: Jalview Version 2 - a multiple sequence alignment editor and analysis workbench. Bioinformatics 2009, 25(9):1189-1191.

62. Castresana J: Selection of conserved blocks from multiple alignments for their use in phylogenetic analysis. Mol Biol Evol 2000, 17:540-552.

63. Nei $\mathrm{M}$, Gojobori T: Simple methods for estimating the numbers of synonymous and nonsynonymous nucleotide substitutions. Mol Biol Evol 1986, 3(5):418-426.

64. Ziheng Y: PAML 4: Phylogenetic Analysis by Maximum Likelihood. Mol Biol Evol 2007, 24(8):1586-1591.

65. Yang Z, Nielsen R, Goldman N, Pedersen A: Codon-substitution models for heterogeneous selection pressure at amino acid sites. Genetics 2000 155(1):431-449.

66. Fares $\mathrm{M}$, Byrne $\mathrm{K}$, Wolfe $\mathrm{K}$ : Rate asymmetry after genome duplication causes substantial long-branch attraction artifacts in the phylogeny of Saccharomyces species. Mol Biol Evol 2006, 23(2):245-253.

67. Yang Z, Wong WNR: Bayes empirical Bayes inference of amino acid sites under positive selection. Mol Biol Evol 2005, 22:1107-1118.

doi:10.1186/1471-2180-12-206

Cite this article as: Vollan et al.: In silico evolutionary analysis of

Helicobacter pylori outer membrane phospholipase A (OMPLA). BMC

Microbiology 2012 12:206.

\section{Submit your next manuscript to BioMed Central and take full advantage of:}

- Convenient online submission

- Thorough peer review

- No space constraints or color figure charges

- Immediate publication on acceptance

- Inclusion in PubMed, CAS, Scopus and Google Scholar

- Research which is freely available for redistribution 